\title{
La biopsia intraoperatoria en patología oncológica urológica
}

\author{
Algaba $\mathrm{F}^{* * *}$, Arce $\mathrm{Y}^{*}$, Santaularia $\mathrm{JM}^{\mathrm{a} *}$, Villavicencio Mavrich H.**** \\ *Sección de Patología. Fundación Puigvert. Barcelona. **Departamento de Ciencias Morfológicas de la \\ Facultad de Medicina de la Universitat Autónoma de Barcelona (UAB). ***Director del Servicio de Urología de \\ la Fundación Puigvert. Barcelona.
}

Actas Urol Esp. 2007;31(9):945-956

\section{RESUMEN}

LA BIOPSIA INTRAOPERATORIA EN PATOLOGÍA ONCOLÓGICA UROLÓGICA

Las indicaciones de la biopsia intraoperatoria (BI) en las cirugias por neoplasias urológicas son muy concretas, esto explica que sólo representen un $7^{\prime} 3 \%$ de las BI en los hospitales generales y motiva que se conozca de una forma precisa su utilidad, siendo éste el motivo la revisión que presentamos.

En términos generales no está justificada la BI para identificar la naturaleza de una masa tumoral, a excepción de: 1) Las masas renales con dudas sobre el origen parenquimatoso o en vías urinarias. 2) En las neoplasias testiculares cuando se plantea la posibilidad de un tratamiento conservador. 3) La determinación de la presencia de un adenocarcinoma de próstata en un donante de órganos con PSA sérico elevado, pero incluso en estas circunstancias hay amplia discusión sobre su necesidad.

La determinación intraoperatoria de los márgenes quirúrgicos es especialmente útil en: 1) Las nefrectomías parciales (puede limitarse a la inspección habiendo pintado con tinta china el margen y sólo muy ocasionalmente se precisa congelación del lecho). 2) El uretral en mujeres con cistectomías totales y sustitución ortotópica. 3) En las penectomías parciales (estudiando siempre el margen uretral y el de los cuerpos cavernosos y esponjoso).

El estudio de los ganglios es un tema ampliamente debatido y salvo en casos del hallazgo de un inesperado aumento del tamaño ganglionar no está indicada la BI sistemática ni en vejiga ni en próstata. Otra es la situación en el carcinoma de pene ya que en los grupos de riesgo intermedio y alto de metástasis ganglionar, aunque haya de un $16 \%$ a un $18 \%$ de falsos negativos se recomienda BI, especialmente de los ganglios centinelas marcados con radioisótopos.

Palabras clave: Biopsia intraoperatoria. Biopsia intraoperatoria tumores renales. Biopsia intraoperatoria tumores vejiga. Biopsia intraoperatoria tumores testículo. Biopsia intraoperatoria cáncer de pene. Biopsia donantes de órganos.

\section{ABSTRACT}

\section{FROZEN SECTION IN UROLOGICAL ONCOLOGY}

The indications of Frozen section diagnosis (FS) in surgery due to urologic neoplasia are quite specific, and this explains the fact that they amount to a mere $7.3 \%$ of the FSs performed in general hospitals. This also makes the precise knowledge of their usefulness necessary, and thus we are submitting the present review.

Generally speaking, FS is not warranted to identify the nature of a tumoral mass, with the following exceptions: 1) Renal masses of a doubtful parenchymal origin, or in the urinary tract: 2) In testicular neoplasias, when the possibility of a conservative treatment arises; 3) Determination of the presence of a prostate adenocarcinoma in an organ donor with high serum PSA; but even in these circumstances its need is widely controversial.

Intraoperative determination of surgical margins is particularly useful in: 1) Partial nephrectomies (it may be limited to inspection after dyeing the margin with Indian ink - bed freezing is very seldom needed); 2) Urethral margins in women with total cystectomies and orthotopic substitution; 3) In partial penectomies (always studying the urethral margin and the cavernosal and spongiosal corpora margins).

The study of the nodes is a widely debated issue, and except for those cases in which unexpectedly increased node size is found, systematic FS is indicated neither of the bladder nor of the prostate. The situation regarding penis carcinoma is different, as in the groups with intermediate and high risk of node metastasis, even though there is around $16 \%-18 \%$ of false negatives FS is recommended, particularly of radioisotope-marked sentinel nodes.

Keywords: Frozen section. Renal cell tumors frozen section. Bladder cancer frozen section. Testicular tumor frozen section. Penile carcinoma frozen section. Frozen section and organ donors. 


\section{FUNCIÓN, INDICACIONES Y LIMITACIONES DE LAS BIOPSIAS INTRAOPERATORIAS}

La indicación de una biopsia intraoperatoria (BI) nace de la necesidad del cirujano mientras está operando, y ésta obedece a una situación de duda durante el acto quirúrgico, de cuya resolución va a depender el curso de la intervención quirúrgica. Ello significa que no ha de pedirse por mera curiosidad del cirujano, ni para poder dar al paciente una información aparentemente definitiva del resultado final.

De acuerdo con lo dicho las principales indicaciones de una BI son de diagnóstico, evaluación de márgenes del tumor y confirmación de que se está tomando suficiente tejido para un diagnóstico diferido.

El estudio patológico durante la cirugía condiciona la toma de decisiones diagnósticas rápidas por parte del patólogo, para ello no sólo ha de tener una buena formación en su especialidad, sino que también ha de tener conocimientos de clínica quirúrgica (qué técnica se está haciendo, qué es lo que el cirujano desea en el momento de hacer la biopsia),

Son muy diversos los tipos de BI. En ocasiones sólo se recibe un pequeño fragmento de la lesión y en este caso se estudia en su totalidad. En otros casos se llega a recibir la totalidad de la pieza y es el patólogo quien escoge la zona más representativa para responder a la pregunta del cirujano.

Diversos factores pueden influir en la interpretación de la BI. Un 9־5\% de los errores, en BI de cirugía general, lo son por un mal muestreo ${ }^{1}$, por lo que una clara comunicación entre los Departamentos de Cirugía y Patología puede disminuir este tipo de error. Las características de la misma técnica (congelación del tejido) justifica que algunas patologías en las que el detalle celular ha de ser más fino queden más perjudicadas que otras, tal es el caso de los linfomas ${ }^{1}$. Por otra parte la capacidad de profundizar en el tejido escogido también queda reducida, haciéndose menos secciones de las muestras y por ello alrededor de una cuarta parte de los infradiagnósticos lo son por esta causa ${ }^{1}$. A todas estas contingencias hemos de añadir las limitaciones en la preparación técnica del material y en la interpretación ${ }^{2}$.
A pesar de lo dicho las discordancias entre el diagnostico intraoperatorio y el definitivo, con verdadera repercusión para el paciente, son escasas, ya que la concordancia entre ambos diagnósticos es de alrededor del 97-98\% $\%^{2,3}$. Sin embargo el nivel de satisfacción con la BI depende de la expectativa que se pone en ella, y al ser un diagnóstico más precario que el diagnóstico postoperatorio es necesario que el cirujano conozca las indicaciones más precisas y las limitaciones de la misma.

A pesar de que la patología urológica oncológica sólo representa alrededor del 7,37\% de los casos de BI en un hospital general ${ }^{3}$, cuando se indica cumple una importante función decisoria ${ }^{4}$ y por ello creemos de gran interés revisar las posibilidades y limitaciones de la BI en urología oncológica, órgano a órgano, siendo éste el objetivo del presente artículo.

\section{MANEJO DE LAS BIOPSIAS INTRAOPERATORIAS}

\section{Manejo en quirófano}

Todas las muestras que se remitan para BI han de ser enviadas en fresco, sin ningún fijador.

Si la pieza es de pequeño tamaño y en el transporte se puede deshidratar es recomendable taparla con una gasa humedecida con suero fisiológico. Está contraindicado el sumergir la pieza en suero fisiológico u otro líquido preservador, ya que el proceso de congelación se dificulta por la gran imbibición hídrica de los tejidos.

Cualquier cuestión o duda que tenga el urólogo ha de estar indicada a través de señales en la pieza, pero lo más aconsejable es que el patólogo haya sido informado y "sensibilizado" previamente a través de la insustituible comunicación urólogo-patólogo.

\section{Manejo en patología}

Una vez ha llegado el tejido a Patología puede procederse de dos formas, o se hace una impronta sobre la muestra (método utilizable en masas tumorales o ganglios linfáticos y no en la evaluación de márgenes) ${ }^{5}$ o se realizan secciones congelando el tejido.

Las secciones congeladas son teñidas con métodos rápidos y aunque no se hacen de rutina estudios inmunohistoquímicos, se están proban- 
do métodos rápidos de esta técnica ${ }^{6}$ y quizás en un futuro próximo se puedan incluir como un procedimiento más. El que pueda restar de la pieza remitida se procesa de forma diferida habitual, conservándose las preparaciones microscópicas usadas para el diagnóstico intraoperatorio.

La eficacia de las improntas y de los cortes por congelación es equiparable, aunque levemente superior para las secciones por congelación (discordancias en un 1,6\% de los diagnósticos por impronta, frente a un $0,8 \%$ en los diagnósticos con secciones en los mismos casos, por estudio simultaneo con ambos métodos) ${ }^{5}$.

\section{Tiempo de demora}

El tiempo de demora entre la toma de la biopsia y la llegada del resultado al cirujano es muy variable, ya que depende de la distancia entre los quirófanos y el servicio de Patología, el método de transporte, el tipo de biopsia (por ejemplo no es lo mismo estudiar una sola muestra que la búsqueda de distintos márgenes de resección), dificultad del caso y forma de comunicar el resultado; por todo ello no puede establecerse un tiempo estándar, aunque en la literatura se refiere un máximo de 20 minutos $^{3}$.

\section{Comunicación del resultado}

Según los diferentes centros la información de la BI se puede hacer oralmente o por escrito, pero sea como sea la forma usada ha de quedar constancia de lo dicho durante el acto quirúrgico y en el dictamen final se ha de reflejar el resultado dado. En caso de una discordancia entre el diagnóstico intraoperatorio y el definitivo es recomendable indicar su causa.

En algunos centros con un gran volumen de biopsias intraoperatorias se establece una unidad de Patología en el área quirúrgica, con lo que los aspectos logísticos son más ágiles.

\section{LA BIOPSIA INTRAOPERATORIA EN LAS NEOPLASIAS RENALES Diagnóstico de la naturaleza de las masas renales}

Hay una cierta discusión sobre la necesidad de determinar intraoperatoriamente la naturaleza de una masa renal que ya se ha decidido intervenir quirúrgicamente, esta discusión acontece por varias razones:
- Por un lado aunque un 16,9\% de masas renales que se extirpan no son carcinomas, sólo un $13 \%$ de ellas $(2,2 \%$ de todas las masas renales) son lesiones en las que se podría discutir la indicación quirúrgica ${ }^{7}$.

- Por otro lado la determinación de la naturaleza de una masa renal incierta durante la cirugía es de una dudosa utilidad, ya que se han comunicado falsos negativos entre el 20 y el $37 \%$ de los $\operatorname{casos}^{8}$, con cifras de falsos positivos muy variables ya que oscilan desde prácticamente inexistentes ${ }^{9}$ hasta un $34 \%^{8}$. Múltiples pueden ser las causas de los falsos negativos: la no representatividad de la muestra por ser demasiado superficial y no llegar a la tumoración propiamente dicha, la ausencia de neoplasia viable (necrosis o fibrosis), la naturaleza quística de la masa ${ }^{10}$ y la dificultad de conservar por congelación la típica arquitectura citológica de gran parte de los carcinomas. El origen de los falsos positivos pueden deberse a la sobre valoración de túbulos aplastados que pueden imitar un tumor ${ }^{11}$, así como a las limitaciones intrínsecas del método de congelación que no permiten una identificación precisa del tamaño nuclear, la diferenciación entre neoplasia y ciertos procesos inflamatorios, así como un correcto diagnóstico diferencial de las lesiones fusocelulares (Fig. 1).

- Y por último vale la pena resaltar que aunque al principio de las nefrectomías parciales parecía ser muy preciso constatar que estuviéramos frente a un carcinoma de baja agresividad ${ }^{12}$, cada día se amplia más la indicación de cirugía conservadora, por lo que no es requerida la participación del patólogo.

Así pues no parece indicado el considerar como un buen método la BI en los carcinomas del parénquima renal. Sólo en los casos en los que el urólogo precise distinguir un carcinoma del parénquima renal con extensión a la vía urinaria de un carcinoma urotelial con invasión del parénquima renal, (distinción que puede comportar cambiar de una nefrectomía a una nefroureterectomía total), el estudio macroscópico y microscópico de la pieza completa de nefrectomía (y no sólo una pequeña muestra del tumor) permitirá al patólogo poder determinar con rapidez el origen de la neoformación ${ }^{13}$. 


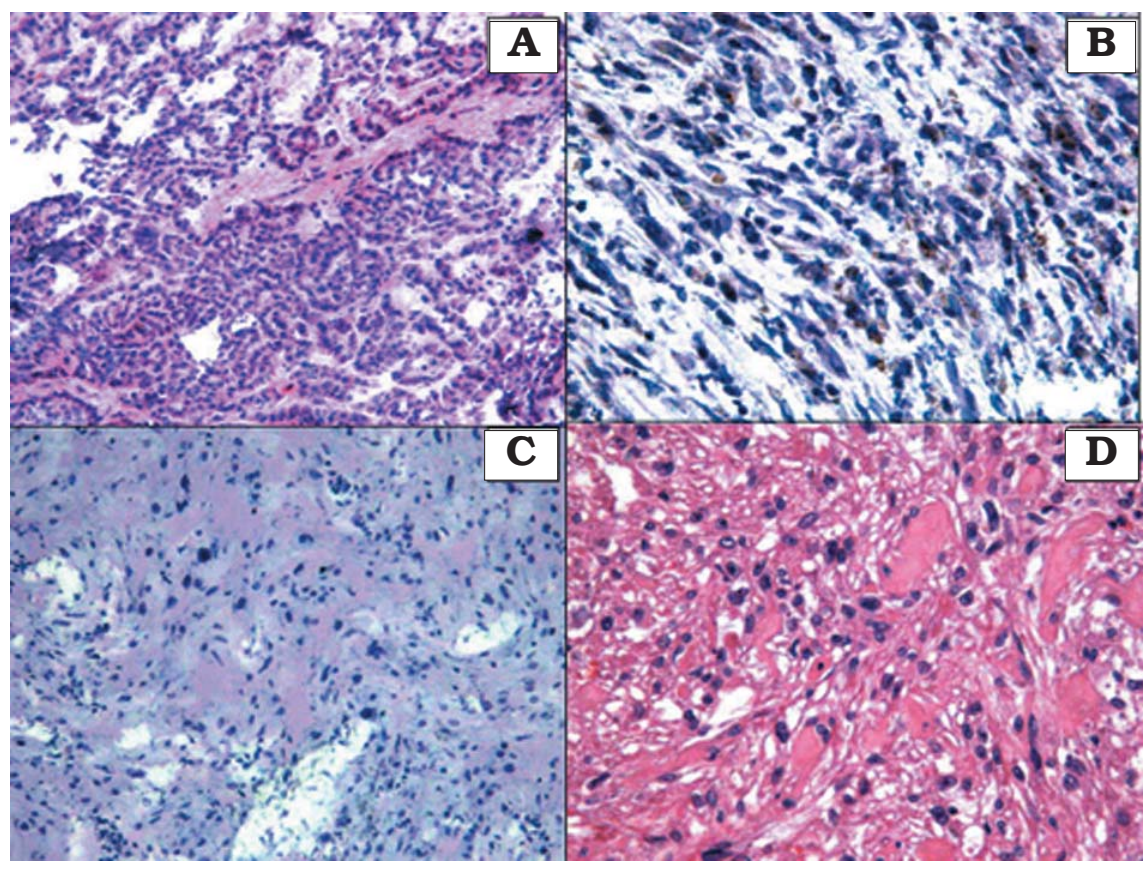

FIGURA 1. La biopsia intraoperatoria en masas renales puede tener dificultades de interpretación. A- Aspecto microscópico de un carcinoma renal papilar (H\&E). BProceso inflamatorio con depósitos de hemosiderina y proliferación de fibroblastos que pueden confundirse con una neoplasia fusocelular. C-Biopsia intraoperatoria de angiomiolipoma de predominio muscular y un aspecto dificil de distinguir de otras lesiones fusocelulares D- El mismo angiomiolipoma en cortes definitivos.

Para situar la discusión hemos de tener presente que el patólogo puede recibir dos tipos distintos de muestras: pequeños fragmentos seleccionados por el urólogo del lecho quirúrgico, o la pieza completa de nefrectomía parcial. Esta segunda pieza evita el aplastamiento del tejido que pueden tener las pequeñas muestras.

La pieza completa de nefrectomía parcial debe ser inspeccionada externamente por el patólogo y el margen quirúrgico pintado con tinta china ${ }^{17}$, seccionada cada $5 \mathrm{~mm}$. y observada la relación entre el tumor y la zona pintada. Si el margen quirúrgico está macroscópicamente distante, con suficiente claridad, del tumor creemos que no es preciso hacer una congelación ${ }^{13}$, ya que no suele añadir ninguna información ${ }^{18}$, sino que incluso

A pesar de ello la literatura anatomo-patológica sigue publicando métodos de identificación rápida de algunos subtipos celulares de carcinoma renal, tal como la metacromasia con azul de toluidina que expresan las células del carcinoma cromófobo, sobretodo en las improntas citológi$\operatorname{cas}^{14}$.

\section{Márgenes de resección en las nefrectomías parciales}

Mucho más útil que determinar intraoperatoriamente la naturaleza de una masa renal es saber el estado de los márgenes quirúrgicos en las nefrectomías parciales.

A pesar de que la causa de la recurrencia local después de una cirugía renal parcial suele ser la multifocalidad subclínica (que a su vez está relacionada con el subtipo celular) ${ }^{15}$, no debe menospreciarse el riesgo que puede suponer un margen quirúrgico afectado, hecho que es más frecuente en los tumores de mayor tamaño ${ }^{16}$. Sin embargo hay discusión en la literatura de cómo actuar, y así mientras que unos autores recomiendan hacer BI sistemáticamente otros dudan de ello. puede confundir ya que se ha referido un $1,3 \%$ de falsos negativos en las secciones congeladas (probablemente por un error de muestreo), y muchas veces la ampliación de la resección por un margen positivo microscópicamente, no se ha acompañado de la demostración de tumor residual ${ }^{19}$.

Para nosotros es cuestionable la opinión de ciertos autores que recomiendan enviar sistemáticamente al patólogo muestras del lecho quirúrgico, incluso con un aspecto normal de la superficie quirúrgica ${ }^{20}$, porque aunque pueden existir áreas de extensión por contigüidad del tumor hacia la "cápsula" tumoral (Fig. 2) se ha demostrado que la distancia (por mínima que sea) desde el tumor al margen quirúrgico libre no comporta ningún riesgo para el enfermo ${ }^{21}$.

En caso de duda siempre debe realizarse la congelación $^{22}$. El margen se considera afectado cuando el tumor alcanza la superficie teñida con la tinta china ${ }^{17}$, pero pueden existir situaciones de difícil interpretación por artefacto quirúrgico (electrocoagulación) por lo que es aconsejable la máxima pulcritud del quirúrgico para no tener falsos positivos por artefacto (Fig. 3). 


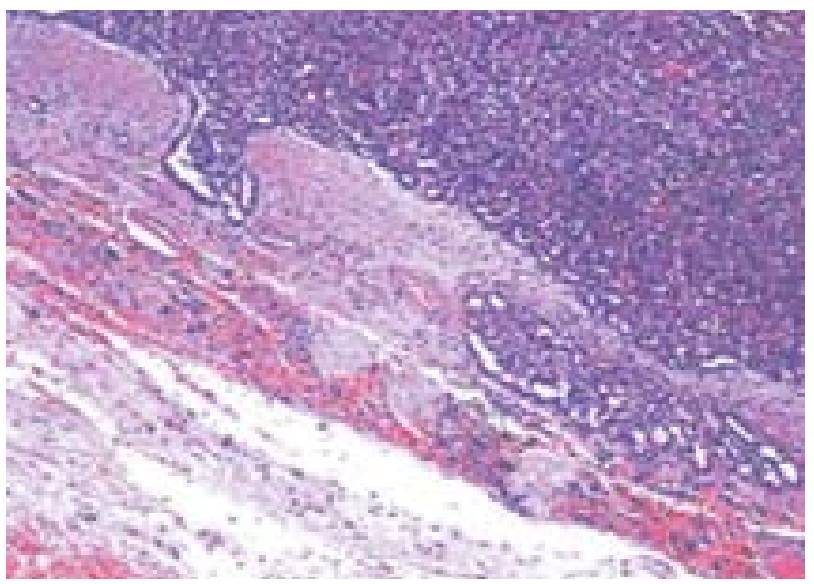

FIGURA 2. Cápsula de un carcinoma renal papilar de tipo 1. Nótese las extensiones de la neoplasia por el espesor de la cápsula, pero sin alcanzar al margen quirúrgico.

La invasión del sistema colector, sin infiltración del margen de sección quirúrgica de la pared de la vía (cáliz), no debe considerarse como un margen positivo ${ }^{23}$.

No existe discusión sobre la necesidad de BI en los casos con sospecha de invasión del tejido perirrenal o invasión ganglionar no detectada antes de la cirugia, ya que condiciona el cambio de la indicación quirúrgica ${ }^{24}$, hecho que no ocurre cuando ya se va a realizar una nefrectomía radical.

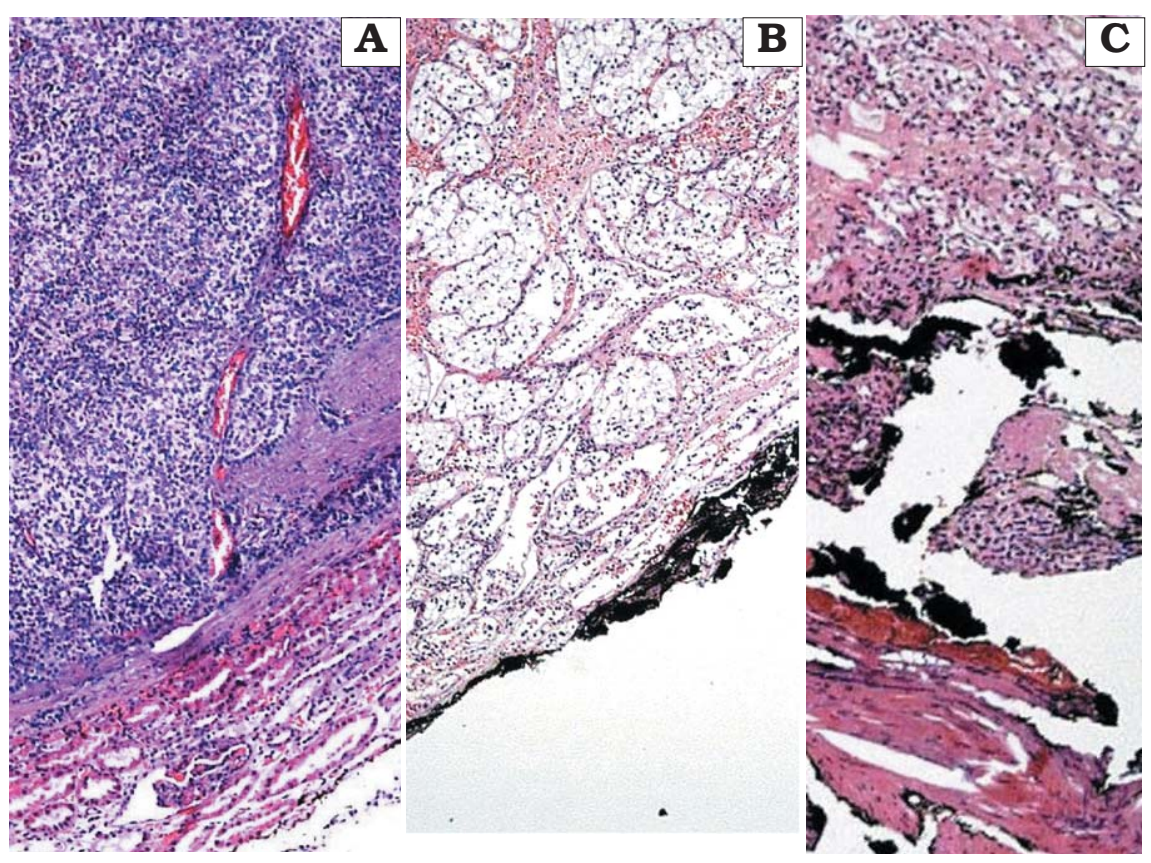

FIGURA3. Los márgenes en las nefrectomias parciales marcados con tinta china: A-Margen quirúrgico negativo. B-Margen quirúrgico positivo. $C$-Margen de difícil interpretación por el artefacto.

\section{LA BIOPSIA INTRAOPERATORIA EN LAS NEOPLASIAS DEL TRACTO URINARIO}

De forma general hay dos situaciones quirúrgicas muy distintas, las cirugias parciales y las radicales. En cada una de ellas hay demandas distintas que van desde el estado de los márgenes, al diagnóstico de la naturaleza de una masa dentro de la vía o de una tumoración externa que la involucra ${ }^{25}$.

\section{Diagnóstico de la naturaleza de los tumores en vía urinaria}

No suele ser requerida la intervención del patólogo intraoperatoriamente, ya que la mayoría de los casos ya han sido categorizados a través de biopsias endoscópicas

Ocasionalmente en los tumores de vías urinarias altas, de difícil acceso, se ha pedido el determinar su naturaleza e incluso el grado de diferenciación. Esta indicación puede ser discutible, pero se fundamenta en la idea de poder hacer resecciones endoscópicas en los tumores de bajo grado (ninguno de nuestros casos de bajo grado invaden la muscular), mientras que en los de alto grado (al ser con más frecuencia invasores de la muscular-75\%) está más indicada una resección reglada por vía abierta. En este tipo de BI las secciones han de ser de la mejor calidad posible, y sólo deben considerarse como altos grados aquellos casos indiscutibles (Fig. 4).

En caso de la identificación histológica de una masa extravesical o extraureteral la situación es equiparable a la de la misma situación en cualquier otra BI.

\section{Márgenes en las resecciones parciales}

Los criterios para indicar una cistectomía parcial son muy estrictos y prácticamente se restringen a los casos de tumores no uroteliales (carcinoma de uraco e invasión focal de la vejiga por una neoplasia extravesical, generalmente del recto-sigma). En 


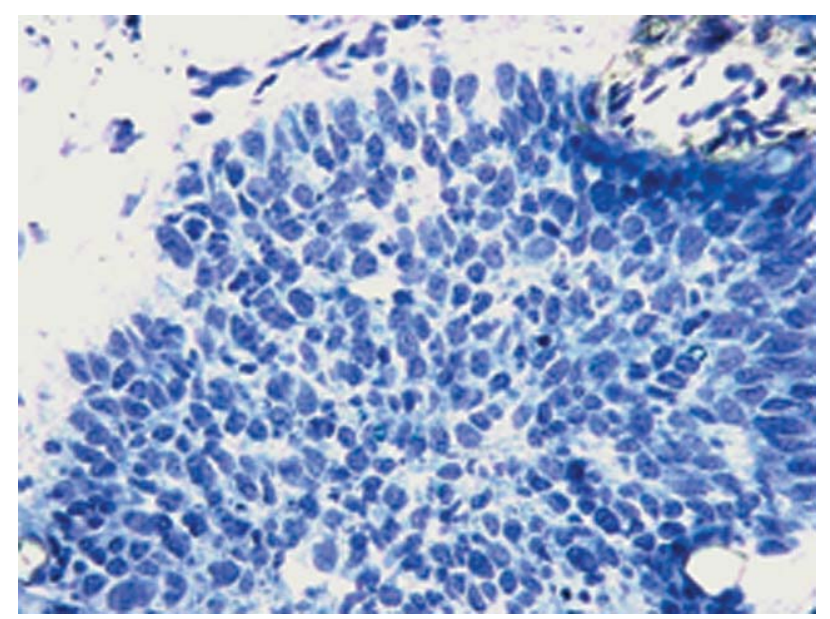

FIGURA 4. Corte por congelación de un carcinoma urotelial de alto grado en vía urinaria alta.

estos casos el urólogo puede remitir muestras de los márgenes para determinar su estado o enviar al patólogo toda la pieza. En este último supuesto ésta debe estar orientada para que el patólogo pueda reconocer la localización de estos márgenes. Los márgenes deben pintarse con tinta china y realizar la selección de las áreas a congelar con una sección perpendicular de todo el espesor de la pared ${ }^{26}$.

La actitud en las piezas de resección parcial de otras localizaciones del tracto urinario es similar a lo descrito en vejiga.

\section{Márgenes en las cistectomias radicales}

Se pueden definir tres márgenes distintos en las piezas de cistectomía total, los ureterales, el uretral y el radial o circunferencial.

- Los márgenes ureterales: Raramente el tumor (como masa) afecta a los márgenes ureterales, y en caso de que el urólogo lo sospeche está indicado el realizar la $\mathrm{BI}$. La situación es totalmente distinta en el caso de que el aspecto macroscópico sea normal, ya que los carcinomas "in situ" son infrecuentes (del 5,7 al 9,5\%) 27,28 , de difícil identificación $^{29}$ (Fig. 5), con un 40\% de falsos positivos ${ }^{28}$, y un significado clínico cuestionable tanto por lo que al riesgo de un carcinoma ulterior en vía urinaria superior se refiere, como para la sobrevida del paciente 27,30 por lo que algunos autores desaconsejan la BI rutinaria de los márgenes ureterales $27,28,30$ ya que el devenir del paciente está más ligado a las características del carcinoma vesical que a la presencia de una neoplasia intraepitelial en los márgenes ureterales ${ }^{27,31}$.

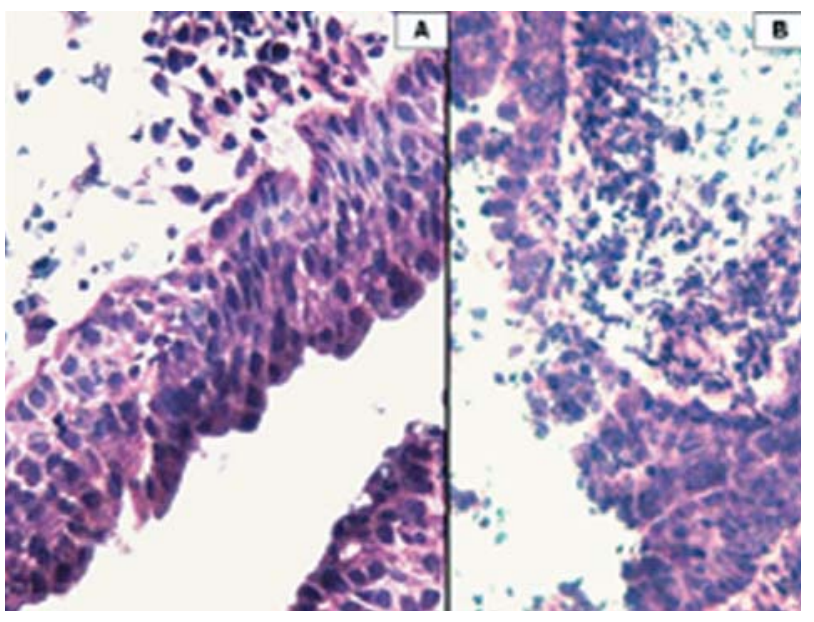

FIGURA. 5. Margen ureteral en cistectomias radicales. Esta figura es para ilustrar la dificultad en distinguir entre un margen normal (A) y un margen con carcinoma "in situ" (B).

- El margen uretral: En la mayoría de los casos el estado de la mucosa uretral es conocido a través de biopsias preoperatorios. A pesar de que hay autores que recomiendan, o prefieren, La BI a la preoperatoria, ya que los resultados de la primera o son iguales a la segunda ${ }^{32}$ o más significativos de la evolución clínica ${ }^{33}$, no es una actitud generalizada ${ }^{34}$. Sin embargo sí que se recomienda $\mathrm{BI}$ uretral sistemática en mujeres a las que se vaya a hacer sustitución vesical ortotópica ${ }^{35}$.

- Margen radial o circunferencial: Como margen radial puede considerarse al tejido adiposo perivesical y el peritoneo. La determinación de su estado debe ser referida en el estudio final ${ }^{26}$, pero sólo se debe realizar BI en caso de que su afectación signifique alguna variación en la cirugia ${ }^{13}$. En el caso de que se tenga que realizar, y al igual que en los otros márgenes ya referidos, debe pintarse con tinta china, para saber siempre la exacta localización del verdadero límite quirúrgico.

- Una situación muy singular son las cistectomías con preservación de la zona periférica de la próstata. Esta indicación quirúrgica es discutida y sobrepasa los limites de esta revisión, sin embargo sí que vale la pena reflejar aquí que los autores que la han realizado han practicado estudios de BI del tejido de la resección transuretral (RTU) de la zona de transición de la próstata, que incluye a la uretra, así como de la "cápsula quirúrgica” (zona periférica) ${ }^{36}$. 


\section{Estudio de los ganglios linfáticos}

La BI de la linfadenectomía regional está en relación a la actitud que tenga el centro con respecto a las metástasis ganglionares.

Si nos guiamos por la sobrevida vemos que no hay supervivencia más allá de los 3 años en los pacientes con enfermedad ganglionar macroscópica, mientras que cuando sólo es microscópica la sobrevida puede ser del 55\% a los 5 años ${ }^{37}$; estas cifras justifican que sólo se contemple la posibilidad de no realizar la cistectomía con ganglios linfáticos positivos macroscópicamente (en cuyo caso la BI está indicada para confirmarlo), mientras que cuando los ganglios son de aspecto normal no es recomendable la $\mathrm{BI}$, ya que no estaría contraindicada la cistectomía.

\section{LA BIOPSIA INTRAOPERATORIA EN LOS CARCINOMAS PROSTÁTICOS Diagnóstico intraoperatorio de adenocarcinoma prostático}

Cuatro son los tipos de muestra que se suelen obtener de las próstatas: los cilindros por aguja de biopsia, los fragmentos de las RTU, las piezas de adenomectomía y las piezas de prostatectomía radical.

En caso de necesitar un estudio intraoperatorio, para determinar la presencia de un eventual adenocarcinoma, se desaconseja el obtener el tejido por aguja de biopsia ya que es una muestra de difícil manejo, escasa representatividad y el tejido que pueda restar tiene importantes artefactos que lo hacen inservible para cualquier estudio ulterior ${ }^{13}$.

La determinación casi sistemática del PSA sérico, con sus consecuentes biopsias si sale elevado y la casi total desaparición de la orquiectomía como tratamiento del cáncer de próstata, ha condicionado que casi nunca el urólogo pida BI de los fragmentos de RTU prostática. Sin embargo si las circunstancias específicas de un paciente concreto motivan su necesidad, el urólogo debe seleccionar los fragmentos que va a remitir (no se pueden congelar todos los fragmentos). Los criterios de selección son poco precisos pero la consistencia mayor de lo normal, y la coloración amarilla pueden orientar hacia carcinoma, con una especificidad del 100\% y una sensibilidad del
$39 \% 38$. Así y todo, las limitaciones son muy grandes, especialmente en los patrones 1 y 2 de Gleason, especialmente si el paciente está con deprivación hormonal, o está bajo el efecto de ciertos tratamientos farmacológicos de la hiperplasia benigna de la próstata que pueden inducir cambios morfológicos similares a los provocados por la hormonoterapia ${ }^{39}$, por lo que se desaconseja el realizar BI en material de RTU.

Cada vez se realizan menos cirugías por vía abierta para el tratamiento de la hiperplasia prostática, por lo que la situación del hallazgo de un área indurada en la cápsula quirúrgica (que corresponde a la zona periférica de la próstata) es infrecuente. Evidentemente que cuando surge una de estas situaciones el conocer la naturaleza de esta porción endurecida puede ser una indicación de BI.

Una situación muy especial la constituye el determinar la presencia de adenocarcinoma de próstata en un donante de órganos con PSA elevado. En esta circunstancia tampoco se han de tomar biopsias por aguja, sino que se debe remitir la próstata entera y el patólogo tomará muestras preferentemente de la zona periférica y externa del órgano ${ }^{13}$. Si no se encuentre un carcinoma con este método se acepta que en caso de que hubiera un carcinoma éste sería tan pequeño que no tendría trascendencia clínica ${ }^{40}$.

\section{Márgenes en las prostatectomías radicales}

Debido a la mejor selección de los pacientes candidatos a una prostatectomía radical no suele ser requerida la participación del patólogo durante la cirugía, sin embargo la disminución de la edad de los pacientes a los que se les diagnostica de cáncer de próstata sí que ha incrementado el número de casos en los que se trata de preservar los plexos vasculonerviosos, y es en estos casos en los que puede ser necesaria la BI de los márgenes.

La manipulación del tejido en Patología cambia según la muestra que se envíe. Si se remite el tejido de los plexos vasculonerviosos el patólogo sólo ha de llevar a cabo la congelación de ellos e interpretarlos. Si se remite la pieza de prostatectomía completa algunos autores recomiendan que el urólogo marque, preferentemente con tinta china para evitar la distorsión anatómica, el área sospechosa, ya que no se puede estudiar todo el margen de la pieza ${ }^{41}$. 
Los márgenes con mayor riesgo de ser positivos cambian según la vía de abordaje quirúrgico, y así se ha observado que el ápex es la porción con más riesgo de estar afectada en las prostatectomías radicales por vía retropúbica, el cuello vesical por vía perineal y las áreas póstero-laterales en la técnica de laparoscopia ${ }^{42}$.

De acuerdo a estos hallazgos hay que prestar una especial atención a los plexos vasculonerviosos en las prostatectomías por vía laparoscópica, sin embargo siempre que se planee una prostatectomía radical con preservación nerviosa (sea cual sea la vía de abordaje) ha de recordarse que la ruta principal de extensión extraprostática es precisamente a través de dichos plexos vasculonerviosos ${ }^{43}$, y por ello en los casos con factores de riesgo de extensión extraprostática, a los que se quiera hacer una cirugía conservadora de los nervios, es recomendable la $\mathrm{BI}$ de ellos, método que ha demostrado su utilidad al tener un valor predictivo positivo de un $73 \%$ y negativo de un $94 \%{ }^{41}$, por lo que se consigue una perfecta selección de los casos a los que se hace preservación de los plexos, sin aumentar la prevalencia de los márgenes positivos ${ }^{44}$.

Sin embargo lo más importante al hallar un margen quirúrgico positivo, sobretodo en la próstata con escasas posibilidades de ampliar la resección, es saber si significa que hay enfermedad residual, y en este aspecto la mayoría de las publicaciones señalan que muy pocos enfermos con BI positiva tienen enfermedad residual en la celda prostática, cosa fácil de entender ya que en pormenorizados estudios de las piezas de prostatectomía radical sólo sutiles aspectos del margen positivo (magnitud del margen afectado e irregularidad) (Fig. 6) son indicativos de un riesgo de cáncer residual $^{45}$, características muy dificiles de definir por congelación rápida. Por lo que se pone en duda el uso sistemático de la $\mathrm{BI}$ de los márgenes ${ }^{46-}$ 48 , salvo en ocasiones concretas como la ya descrita de la prostatectomía con preservación vasculonerviosa o con áreas sospechosas a la palpación halladas durante la cirugía ${ }^{49}$.

\section{Estudio de los ganglios linfáticos}

Los nomogramas de predicción construidos por la combinación de las cifras de PSA sérico, del "score" Gleason de la biopsia y la categoría $\mathrm{T}$ (clinica) ${ }^{50}$ tienen una alta sensibilidad en determinar los pacientes con bajo, intermedio o alto riesgo de metástasis ganglionar.

La rentabilidad de la BI es muy alta en ganglios macroscópicamente sospechosos, pero en casos de micrometástasis hay hasta un 19\% de casos falsos negativos ${ }^{51}$. Por ello la indicación de la BI de los ganglios linfáticos es cuestionable y depende fundamentalmente de la actitud del urólogo. Los pacientes con riesgo de metástasis bajo e intermedio no se benefician de la BI y sólo en los enfermos que a pesar del alto riesgo el urólogo cree indicado en intento de una prostatectomía radical puede estar justificada la BI para evitar innecesaria morbilidad, si se confirman las metástasis ganglionares ${ }^{52}$. En estos casos si el urólogo constata un ganglio aumentado de tamaño o consistencia sólo debe remitir dicho ganglio al patólogo, si no hay ningún nódulo detectable se remite el tejido adiposo de la zona ganglionar.

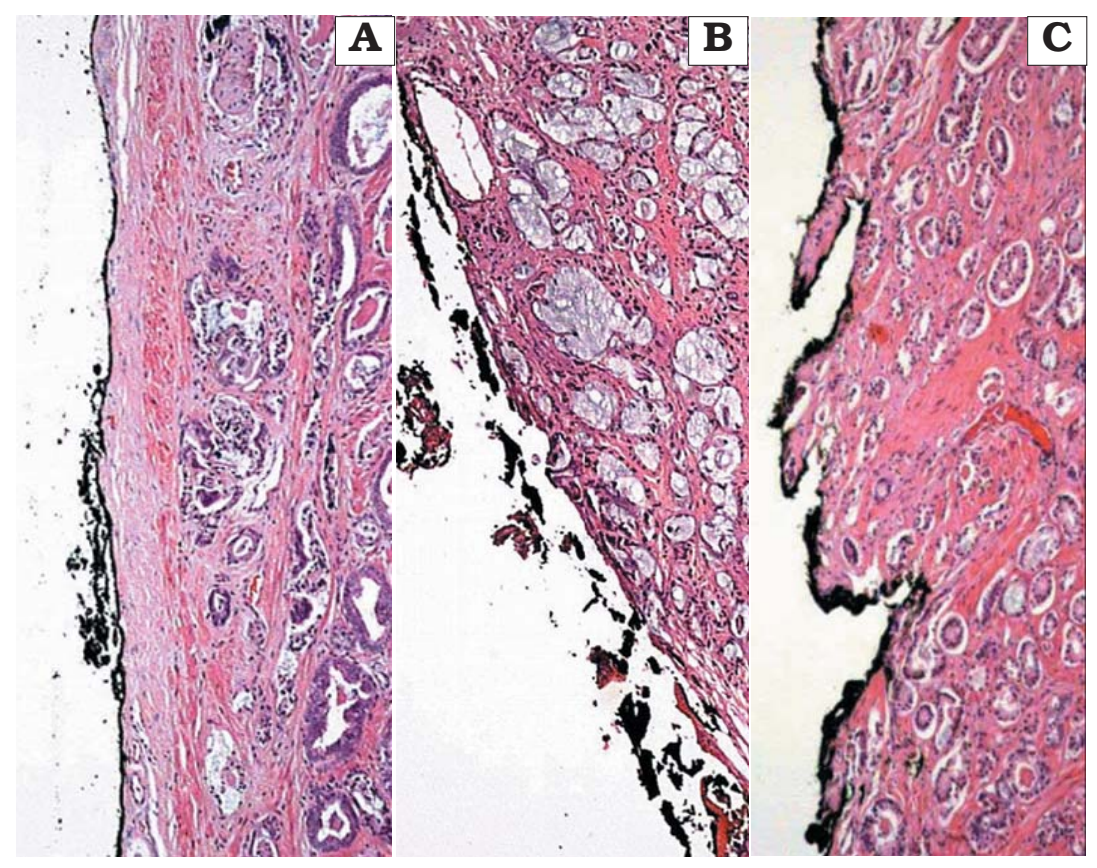

FIGURA. 6. Los márgenes en las prostatectomias radicales. A- Margen negativo. B) Margen positivo de superficie lisa. C) Margen positivo de superficie irregular. Estas imágenes son de muestras procesas en parafina, pero sirven para ilustrar el grado de escrupulosidad con el que han de ser valorados los márgenes y nos ayudan a entender que esta calidad es dificil de obtener en cortes por congelación. 
Es recomendable que el patólogo conozca el score Gleason de la biopsia para optimizar la selección de que porción del tejido se ha de congelar, y así se recomienda que en los casos con score 8 o superior se intenten congelar todos los nódulos hallados en la disección ${ }^{53}$, mientras que si es inferior con sólo estudiar de dos a tres ganglios se puede hallar más del $50 \%$ de las micrometásta$\mathrm{sis}^{54}$. Así y todo la práctica de la BI en las prostatectomías radicales no está recomendada como un método de rutina ya que los parámetros prequirúrgicos son muy eficientes ${ }^{55,56}$.

Sin contradecir lo dicho hasta ahora puede ser que en un próximo futuro sea requerida la BI de ganglios centinelas marcados con radioisótopos, con la idea de extender las linfadenectomía locales de ciertos carcinomas ${ }^{57,58}$.

\section{LA BIOPSIA INTRAOPERATORIA EN LOS TUMORES DEL TESTÍCULO}

Las metodologías diagnósticas actuales de las masas tumorales en testículo definen en un alto porcentaje su naturaleza, por este motivo es muy infrecuente que el patólogo sea requerido intraoperatoriamente para diagnosticarlas. Sin embargo la actitud conservadora actual, las tumoraciones en testículos únicos o masas tumorales bilaterales, la sospecha de una neoplasia no germinal (especialmente en niños), así como el hallazgo incidental ecográfico de una masa testicular no palpable, pueden llevar al urólogo a pedir una $\mathrm{BI}^{59}$.

A pesar de que pueden haber problemas de diagnóstico diferencial entre los seminomas y los procesos inflamatorios granulomatosos, así como entre teratoma y metaplasia escamosa de los hidroceles ${ }^{60}$, la fiabilidad de la BI de las masas testiculares es alta, ya que pueden distinguirse prácticamente todas las neoplasias benignas de los tumores germinales tanto en adultos ${ }^{61-63}$ como en los niños, en los que hasta un $50 \%$ de los tumores son benignos ${ }^{64}$; sin embargo es opinión de algunos autores que no es obligado el realizar una BI ni en los tumores benignos, ni en los tumores germinales, si antes se ha tomado la decisión de cirugía conservadora según las estrictas directrices consensuadas $^{65}$, ya que en el peor de los casos siempre se puede realizar la orquiectomía diferida ${ }^{66}$, eventualidad que ocurre raramente. Lo que sí que debe hacerse sistemáticamente es tomar muestras del parénquima testicular normal peritumoral para realizar estudio en parafina (no por congelación) y detectar posibles cambios de neoplasia intratubular de células germinales vecinas a la neoplasia ${ }^{67}$, lo que condicionaría un tratamiento ulterior.

\section{LA BIOPSIA INTRAOPERATORIA EN EL CÁNCER DE PENE \\ Diagnóstico intraoperatorio del cáncer de pene}

Está totalmente desaconsejada la BI de una masa o úlcera peneana para establecer la naturaleza de la lesión, ya que hay carcinomas escamosos muy bien diferenciados que pueden parecer lesiones benignas ${ }^{68,69} \mathrm{y}$, viceversa, algunos procesos hiperplásicos pseudoepiteliomatosos que pueden parecer carcinomas ${ }^{70}$. Por lo tanto todo enfermo al que se le vaya a tratar una lesión peneana ésta se ha de categorizar previamente con la biopsia prequirúrgica.

\section{Márgenes en las piezas de penectomía}

Prácticamente todas las penectomías son remitidas al patólogo para que evalúe durante la intervención quirúrgica los márgenes de resección.

A pesar de que de una forma clásica siempre se ha indicado que para ser un buen margen de resección la neoplasia debe distar al menos $2 \mathrm{~cm}$ de él, recientes estudios han demostrado que con distancias milimétricas ya es suficientemente seguro ${ }^{71}$.

Para un correcto estudio de los márgenes se han de conocer las vías de diseminación (Fig. 7), de acuerdo con ellas quedan definidos los siguientes:

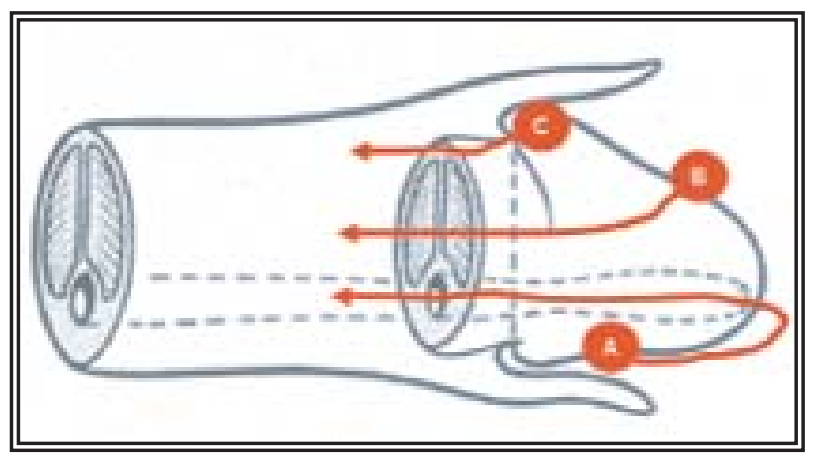

FIGURA. 7. Vías de diseminación hacia los márgenes de resección en la penectomia parcial. 
- Margen uretral. Siempre ha de ser estudiado, incluso en ausencia de lesión macroscópica, y especialmente en los carcinomas con crecimiento por extensión superficial. Puede ser remitido al patólogo aisladamente, y si se recibe con la pieza de exéresis puede estar a otro nivel que el margen del tejido de los cuerpos cavernosos y esponjoso (depende del tipio de reconstrucción que realice el urólogo) ${ }^{72}$.

- Márgenes de los cuerpos cavernosos y cuerpo esponjoso también ha de ser estudiados sistemáticamente, sobretodo en los carcinomas de crecimiento vertical ${ }^{73}$.

- Margen de la fascia del pene. Esta es una porción de especial interés en los carcinomas escamosos de inicio o extensión al surco balanoprepucial.

- Margen cutáneo. Raramente es necesario estudiarlo salvo en los infrecuentes casos de carcinoma de pene originado en el cuerpo peneano.

- Márgenes en las resecciones del prepucio. En el carcinoma de prepucio se han de estudiar, en todo su grosor, el margen circunferencial ${ }^{72}$.

- Márgenes en las penectomias totales. Sólo deben ser estudiados la uretra y los tejidos blandos de alrededor ${ }^{72}$.

\section{Estudio de los ganglios linfáticos}

La aproximación a la BI de los ganglios linfáticos se rige por el grupo de riesgo al que pertenezca el paciente y a la presencia de nódulos palpables o no, y así:

- En los pacientes de bajo riesgo sin nódulos palpables no se recomienda la realización de $\mathrm{BI}^{74}$.

- En los enfermos de riesgo intermedio sin nódulos palpables se recomienda la $\mathrm{BI}$, siendo en esta situación donde la discusión del ganglio centinela tiene más importancia, ya que después del olvido del ganglio de Cabañas (ganglio centinelas estático) actualmente se vuelve a valorar la posibilidad de buscar el ganglio centinela marcado con radioisótopos (ganglio centinela dinámico), y así hay autores que recomiendan esta última forma de ganglio centinela ${ }^{75}$ aunque hay un porcentaje del $16 \%$ al $18 \%$ de falsos negativos ${ }^{76,77}$; así y todo otros autores siguen considerando que se ha de seguir con la linfadenectomía inguinal superficial y modificada con $\mathrm{BI}^{77}$.
- En enfermos con elevado riesgo de enfermedad metastática ganglionar se recomienda la BI para poder realizar linfadenectomía según el resultado ${ }^{78}$.

- Cuando los ganglios son palpables la BI puede definir la naturaleza (inflamatoria o neoplásica) de la adenomegalia.

Todo lo referido hasta ahora es un reflejo de la experiencias recogida en la literatura, así como de la obtenida por los años de interrelación con los urólogos de nuestro centro, y ésta es precisamente la conclusión que creemos más importante. Si ya es imprescindible para una buena patología la interrelación urólogo-patólogo, patólogourólogo, ésta es fundamental para la optimización del resultado en las biopsias intraoperatorias. El preciso conocimiento de lo qué quiere decir el urólogo y el patólogo, así como lo que están viendo cada uno de ellos en el momento quirúrgico permite una buena comunicación interpersonal, base de cualquier buen hacer del ser humano.

\section{REFERENCIAS}

1. Rogers C, Klatt EC, Chandrasoma OP. Accuracy of frozensection diagnosis in a teaching hospital. Arch Pathol Lab Med. 1987;111(6):514-517.

2. Evans CA, Suvarna SK. Intraoperative diagnosis using the frozen section technique. J Clin Pathol 2006;59(3):334.

3. Bailón E, Guzmán F, Petit A, Carreras J, Nadal A, Ribalta T. Concordancia diagnóstica en biopsias peroperatorias (intraoperatorias). Evaluación de 1560 casos estudiados en el Hospital Clinic de Barcelona. Comunicación al VII Congrés de 1,Associació catalana d,Anatomia Patológica (ACMB) Andorra la Vella 2006: 103-104.

4. Giessing M, Rudolph B, Loening SA. Relevance of intraoperative frozen section diagnostics in urological surgery. Aktuelle Urol. 2005;36(6):512-518.

5. Guarda LA: Intraoperative cytologic diagnosis: evaluation of 370 consecutive intraoperative cytologies. Diagn Cytopathol. 1990;6(4):235-242.

6. Johnston EI, Beach RA, Waldrop SM, Lawson D, Cohen C. Rapid intraoperative immunohistochemical evaluation of sentinel lymph nodes for metastatic breast carcinoma. Appl Immunohistochem Mol Morphol. 2006;14(1):57-62.

7. Silver DA, Morash C, Brenner P, Campbell S, Russo P. Pathologic findings at the time of nephrectomy for renal mass. Ann Surg Oncol 1997; 4(7): 570-574.

8. Dechet CB, Sebo T, Farrow G, Blute MI, Engen DE, Zincke H. Prospective analysis of intraoperative frozen needle biopsy of solid renal masses in adults. J Urol. 1999;162(4): 1282-1284.

9. Cloix P, Martin X, Pangaud C, Maréchal J, Bouvier R, Barat $\mathrm{D}$, et al. Surgical management of complex renal cysts: a series of 32 cases. J Urol. 1996;156(1):28-30.

10. Bielsa O, Lloreta J, Gelabert-Mas A. Cystic renal cell carcinoma: pathological features, survival and implications for treatment. Br J Urol. 1998; 82(1):16-20. 
11. McHale T, Malkowicz SB, Tomazewski JE, Genera EM. Potential pitfalls in the frozen section evaluation of parenchymal margins in nephron-sparing surgery. Am J Clin Pathol 2002;118(6):903-910.

12. Krejci KG, Blute ML, Cheville JC, Sebo TJ, Lohse CM, Zincke H. Nephron-sparing surgery for renal cell carcinoma: clinicopathologic features predictive of patient outcome. Urology. 2003;62(4):641-646.

13. Algaba F, Arce Y, Lopez-Beltran A, Montironi R, Mikuz G, Bono AV; European Society of Uropathology; Uropathology Working Group. Intraoperative frozen section diagnosis in urological oncology. Eur Urol. 2005;47(2):129-136.

14. Shet T, Desai S. Toluidine positivity in chromophobe renal cell carcinoma: use in intraoperative diagnosis. Histopathology. 2005;47(5):534-535.

15. Cheng WS, Farrow GM, Zincke H. The incidence of multicentricity in renal cell carcinoma. J Urol. 1991;146(5): 12211223.

16. Wunderlich H, Reichelt O, Schumann S, Schlichter A Kosmehl H, Werner W, et al. Nephron sparing surgery for renal cell carcinoma $4 \mathrm{~cm}$. or less in diameter: indicated or under treated?. J Urol. 1998;159(5):1465-1469.

17. Algaba F, Trias I, Scarpelli M, Boccon-Gibod L, Kirkali Z, Van Poppel H. Handling and pathology reporting of renal tumor specimens. Eur Urol 2004;45(4):437-443.

18. Kubinski DJ, Clark PE, Assimos DG, Hall MC. Utility of frozen section analysis of resection margins during partial nephrectomy. Urology. 2004;64(1):31-34.

19. Duvdevani M, Laufer M, Kastin A, Mor Y, Nadu A, Hanani J, et al. Is frozen section analysis in nephron sparing surgery necessary? A clinicopathological study of 301 cases. J Urol. 2005; 173(2):385-387.

20. Eroglu M, Unsal A, Bakirtas H, Tekdogan U, Ataoglu O, Balbay MD. Routine frozen-section biopsy from the surgical bed should be performed during nephron-sparing surgery for renal cell carcinoma. Scand J Urol Nephrol. 2005; 39(3):222225.

21. Berdjis N, Hakenberg OW, Zastrow S, Oehlschlager S, Novotny V, Wirth MP. Impact of resection margin status after nephron-sparing surgery for renal cell carcinoma. BJU Int. 2006;97(6):1208-1210.

22. Timsit MO, Bazin JP, Thiounn N, Fontaine E, Chretien Y, Dufour B, et al. Prospective study of safety margins in partial nephrectomy: intraoperative assessment and contribution of frozen section analysis. Urology. 2006;67(5):923-926.

23. Thomas DH, Verghese A, Kynaston HG, Griffiths DFR Analisis of the prognosis implications of different tumour margin types in renal cell carcinoma. Histopathology 2003; 43(4):374-380.

24. Lerner Se, Tsai H, Flanigan RC, Trump DL, Fleischann J. Renal cell carcinoma: considerations for nephron-sparing surgery. Urology 1995; 45(4):574-577.

25. Truong LD, Krishnan B, Shen SS. Intraoperative pathology consultation for kidney and urinary bladder specimens. Arch Pathol Lab Med. 2005;129(12):1585-1601.

26. López-Beltrán A, Bassi PF, Pavone-Macaluso M, Montironi R: Handling and pathology reporting of specimens with carcinoma of the urinary bladder, ureter, and renal pelvis. Eur Urol. 2004;45(3):257-266.

27. Batista JE, Palou J, Iglesias J, Sanchotene E, da Luz P, Algaba F, et al. Significance of ureteral carcinoma in situ in specimens of cystectomy. Eur. Urol. 1994;25(4):313-315.

28. Nakanishi S, Nishiyama H, Ito M, Yoshimura K, Kamoto T, Ogawa O. Management of concomitant ureteral carcinoma in situ at radical cystectomy. Int J Urol. 2006;13(5):524-528.
29. Schoenberg MP, Carter HB, Epstein JI. Ureteral frozen section analysis during cystectomy: a reassessment. J Urol 1996;155(4):1218-1220.

30. Lee SE, Byun SS, Hong SK, Chang IH, Kim YJ, Gill MC, et al. Significance of cancer involvement at the ureteral margin detected on routine frozen section analysis during radical cystectomy. Urol Int. 2006;77(1):13-17.

31. Silver DA, Stroumbakis N, Russo P: Ureteral carcinoma in situ at radical cystectomy does the margin matter?. J Urol. 1997;158(3 Pt 1):768-771.

32. Stein JP, Grossfeld GD, Freeman JA. Orthotopic lower urinary tract reconstruction in women using the Kock ileal neobladder: updated experience in 34 patients. J Urol. 1997;158 (2):400-405.

33. Lebret T, Herve JM, Barre P, Gaudez F, Lugagne PM, Barbagelatta $\mathrm{M}$, et al. Urethral recurrence of transitional cell carcinoma of the bladder. Predictive value of preoperative latero-montanal biopsies and urethral frozen sections during prostatocystectomy. Eur Urol. 1998;33(2):170-174.

34. Murphy WM, Grignon DJ, Perlman EJ. Tumors of the kidney, bladder, and related urinary structures. página 343. AFIP Atlas of tumor pathology Series 4. ARP. Washington 2004.

35. Ali-el-Dein B, Abdel-Latif M, Ashamallah A, Abdel-Rahim M, Ghoneim M. Local urethral recurrence after radical cystectomy and orthotopic bladder substitution in women: a prospective study. J Urol. 2004;171(1):275-278.

36. Vallancien G, Abou El Fettouh H, Cathelineau X, Baumert H, Fromont G, et al. Cystectomy with prostate sparing for bladder cancer in 100 patients: 10-year experience. J Urol. 2002; 168(6):2413-2417.

37. Leissner J, Hohenfellner R, Thuroff JW, Wolf HK. Lymphadenectomy in patients with transitional cell carcinoma of the urinary bladder: significance for staging and prognosis. BJU Int. 2000; 85(7):817-823.

38. Geddy PM, Reid IM. Selective sampling of yellow prostate chips: A specific method for detecting Prostatic adenocarcinoma. Urol Int. 1996;56(1):33-35.

39. Di Silverio F, Monti S, Sciarra S. Effects of long term treatment with Serenoa repens (Permixon) on the concentrations and regional distribution of androgens and epidermal growth factor in benign hyperplasia. Prostate 1998;37(2):77-83.

40. Mikuz G, Montironi R, Lopez-Beltran A, Bussolati G. The dilemma of multiorgan donors with high serum PSA - a pathologist's proposal. Virchows Arch. 2006 Aug;449(2):273276. Epub 2006 May 5.

41. Goharderakhshan RZ, Sudilovsky D, Carroll LA, Grossfeld GD, Marn R, Corroll PR. Utility of intraoperative frozen section analysis of surgical margins in region of neurovascular bundles at radical prostatectomy. Urology 2002;59(5):709714.

42. Salomón L, Anastasiadis AG, Lebrel O, Katz R, Saint F, Taille A, et al. Location of positive surgical margins after retropubic, perineal, and laparoscopic radical prostatectomy for organ-confined prostate cancer. Urology 2003;61(2):386-390.

43. Villers A, McNeal JE, Redwine EA. The role of perineural space invasion in the local spread of Prostatic adenocarcinoma. J Urol. 1989;142(3):763-768.

44. Fromont G, Baumert H, Cathelineau X, Rozet F, Validire P, Vallancien G. Intraoperative frozen section analysis during nerve sparing laparoscopic radical prostatectomy: Feasibility study. J Urol. 2003;170(5):1843-1846.

45. Ojea Calvo A, Gonzalez Pineiro A, Dominguez Freire F, Alonso Rodrigo A, Rodriguez Iglesias B, Benavente Delgado J. Implicaciones pronósticas de los márgenes positivos en las piezas de prostatectomía radical: Actas Urol Esp. 2005;29: 641-656. 
46. Lepor H, Kaci L. Role of intraoperative biopsies during radical retropubic prostatectomy. Urology. 2004;63(3):499-502.

47. Dillenburg W, Poulakis V, Witzsch U, de Vries R, Skriapas K, Altmansberger HM, et al. Laparoscopic radical prostatectomy: the value of intraoperative frozen sections. Eur Urol. 2005;48(4):614-621.

48. Tsuboi T, Ohori M, Kuroiwa K, Reuter VE, Kattan MW, Eastham JA, et al. Is intraoperative frozen section analysis an efficient way to reduce positive surgical margins? Urology. 2005;66(6): 1287-1291.

49. Rapp DE, Orvieto MA, Lucioni A, Gong EM, Shalhav AL, Brendler $\mathrm{CH}$. Intra-operative prostate examination: predictive value and effect on margin status. BJU Int. 2005;96(7): 1005-1008.

50. Partin AW, Catan MW, Subong ENP. Combination of prostate-specific antigen, clinical stage, and Gleason score to predict pathological stage of localized prostate cancer. A multiinstitutional update. A multi-institutional up-date. JAMA 1997;277(18): 1445-1451.

51. Catalona WJ, Stein AJ. Accuracy of frozen section detection of lymph node metastases in prostatic carcinoma. J Urol. 1982;127(3):460-461.

52. Beissner RS, Stricker JB, Speights VO, Coffield KS, Spiekerman AM, Riggs M. Frozen section diagnosis of metastatic prostate adenocarcinoma in pelvic lymphadenectomy compared with nomogram prediction of metastasis. Urology 2002;59(5):721-725.

53. Epstein JI. Pathologic assessment of the surgical specimen. Urol Clin N Amer. 2001;28(3):567-594.

54. Epstein JI, Oesterling JE, Eggleston JC. Frozen section detection of lymph nodes metastases in prostatic carcinoma: Accuracy in grossly uninvolved pelvis lymphadenectomy specimens. J Urol. 1986;136:1234-1237.

55. Epstein JI, Amin M, Boccon-Gibod L, Egevad L, Humphrey PA, Mikuz G, et al. Prognostic factors and reporting prostate carcinoma in radical prostatectomy and pelvic lymphadenectomy specimens. Scand J Urol Nephrol. Supplement 2005; 216:34-63.

56. Montironi R, van der Kwast T, Boccon-Gibod L, Bono AV, Boccon-Gibod L. Handling and pathology reporting of radical prostatectomy specimens. Eur Urol. 2003;44(6):626-636.

57. Jeschke S, Nambirajan T, Leeb K, Ziegerhofer J, Sega W, Janetschek G. Detection of early lymph node metastases in prostate cancer by laparoscopic radioisotope guided sentinel lymph node dissection. J Urol. 2005;173(6):1943-1946.

58. Brenot-Rossi I, Bastide C, Garcia S, Dumas S, Esterni B, Pasquier J, et al. Limited pelvic lymphadenectomy using the sentinel lymph node procedure in patients with localised prostate carcinoma: a pilot study. Eur J Nucl Med Mol Imaging. 2005;32(6):635-640.

59. Winstanley AM, Mikuz G, Debruyne F, Schulman CC, Parkinson MC. Handling and reporting of biopsy and surgical specimens of testicular cancer. Eur Urol. 2004;45(5):564573.

60. Shukla AR, Woodard C, Carr MC, UHF DS, Canning DA, Zderic SA, Kolon TF, Zinder III, H. Experience with testis sparing surgery for testicular teratoma. J Urol. 2004;171(1):161163.

61. Elert A, Olbert P, Hegele A, Barth P, Hofmann R, Heidenreich A. Accuracy of frozen section examination of testicular tumors of uncertain origin. Eur Urol. 2002;41(3):290-293.

62. Connolly SS, D’Arcy FT, Bredin HC, Callaghan J, Corcoran MO. Value of frozen section analysis with suspected testicular malignancy. Urology. 2006;67(1):162-165.
63. Leroy X, Rigot JM, Aubert S, Ballereau C, Gosselin B. Value of frozen section examination for the management of nonpalpable incidental testicular tumors. Eur Urol. 2003; 44(4): 458-460.

64. J.S. Valla for the Group D'Etude en Urologie Pediatrique: Testis-sparing surgery for benign testicular tumors in children. J Urol. 2001;165(6 Pt 2):2280-2283.

65. Albers P, Albrecht W, Algaba F, Bokemeyer C, CohnCedermark G, Horwich A, et al. Guidelines on testicular cancer. Eur Urol. 2005;48(6):885-894.

66. Albers P. Organ-sparing surgery for testicular lesions. Eur Urol supplements 5. 2006:522-524.

67. Manivel JC, Reinberg Y, Niehans GA, Fraley EE. Intratubular germ cell neoplasia in testicular teratomas and epidermoid cysts. Correlation with prognosis and possible biologic significance. Cancer 1989;64:715-720.

68. Seixas AL, Ornellas AA, Marota A, Wisnescky A, Campos F, de Moraes JR. Verrucous carcinoma of the penis: retrospective analysis of 32 cases. J Urol. 1994;152(5 Pt 1):1476-1478.

69. Cubilla AL, Velazquez EF, Young RH. Pseudohyperplastic squamous cell carcinoma of the penis associated with lichen sclerosus, an extremely well-differentiated, nonverruciform neoplasm that preferentially affects the forskin and is frequently misdiagnosed. A report of 10 cases of a distinctive clinicopathologic entity. Am J Surg Pathol. 2004;28(7):895-900.

70. Young RH, Srigley JR, Amin MB, Ulbright TM, Cubilla AL. Tumors of the prostate gland, seminal vesicles, male urethra and penis. pp: 403-488 In Atlas of tumor pathology. $3^{\text {rd }}$ Series ed. Washington DC: Armed Forces Institute of Pathology.2000.

71. Minhas S, Kayes O, Hegarty P, Kumar P, Freeman A, Ralph D. What surgical resection margins are required to achieve oncological control in men with primary penile cancer?. BJU Int. 2005;96(7): 1040-1043

72. Velázquez EF, Soskin A, Bock A, Codas R, Barreto JE, Cubilla AL. Positive resection margins in partial penectomies. Sites of involvement and proposal of local routes of spread of penile squamous cell carcinoma. Am J Surg Pathol. 2004; 28(3): 384-389.

73. Rubio-Briones J, Villavicencio H, Regalado R, Chechile G, Algaba F, Monreal, F, et al. Carcinoma escamoso de pene: Protocolo de tratamiento de acuerdo con una experiencia de 14 años. Arch Esp Urol. 1997;50(5):473-480.

74. Solsona E, Algaba F, Horenblas S, Pizzocaro G, Windahl T. EAU guidelines on penile cancer. Eur Urol. 2004;46(1):1-18.

75. Akduman B, Fleshner NE, Ehrlich L, Klotz L. Early experience in intermediate-risk penile cancer with sentinel node identification using the gamma probe. Urology 2001;58(1):65-68.

76. E Kroon BK, Horenblas S, Meinhardt W, van der Poel HG, Bex A, van Tinteren $\mathrm{H}$, et al. Dynamic sentinel node biopsy in penile carcinoma: evaluation of 10 years experience. Eur Urol. 2005;47(5):601-606.

77. Izawa J, Kedar D, Wong F, Pettaway CA. Sentinel luymph node biopsy in penile cancer: evolution and insights. Can J Urol. 2005 Feb;12 Suppl 1:24-29.

78. d'Ancona CA, de Lucena RG, Querne FA, Martins MH, Denardi F, Netto NR Jr. Long-term followup of penile carcinoma treated with penectomy and bilateral modified inguinal lymphadenectomy. J. Urol. 2004;172(2):498-501.

Correspondencia autor: Dr. F. Algaba Arrea

Sección de Patología. Fundación Puigvert

Cartagena 340-350 - 08025 Barcelona

Tel.: 934169700

E-mail autor: falgaba@fundacio-puigvert.es

Información artículo: Original - Biopsia intraoperatoria 